\title{
DARE VOCE ALLE VITE MARGINALI: PLURILINGUISMO DI GENERE NELLA NARRATIVA DI LAURA PARIANI
}

\author{
Gigliola Sulis \\ University of Leeds, UK
}

This article analyses the literary work of Laura Pariani (195 I-), a writer who, over the last twenty years, has gained a niche position in the contemporary canon. After situating Pariani in the context of Italian fiction in the I990s-20IOs, the analysis focuses on the 'centrality of margins' as the inspiring principle underpinning her production. In Pariani's writings marginality - geographical, gender-based, generational, linguistic - becomes a privileged point of view from which to assess the world. The protagonists of her works are women, but also children, immigrants, the socially marginalized, often dialect speakers, or those caught in situations of opposition to or compromise with the 'language of power', covering a chronological range from the sixteenth century to today. The decision to place those whom History has ignored at the centre of the stories, to give voice to the voiceless, is investigated here with a focus on multilingualism, on the places of narration, and on narrative structures, and is related back to the author's youthful militancy in the youth movements of the I970s, including Milan's feminist collectives.

KEYWORDS: Laura Pariani, marginality, multilingualism, Italian fiction in the I990s and 2000s, women writing, contemporary canon

\section{'SCRITTORI VERTICALI' DEGLI ANNI NOVANTA-DUEMILA}

Dopo la progressiva crisi del romanzo degli anni Sessanta e Settanta del Novecento, il ritorno alla narrativa degli anni Ottanta e della prima metà dei Novanta è caratterizzato, nella letteratura mainstream italiana, dal postmoderno internazionalizzante di scrittori come Italo Calvino, Umberto Eco, Andrea De Carlo, Daniele Del Giudice, Antonio Tabucchi, Alessandro Baricco. ${ }^{{ }^{\top}}$ Pur se disuguali sotto molti punti di vista, gli autori citati sono accomunati da almeno due elementi: la preferenza per narrazioni cosmopolite - storie di un'Italia postmoderna che si è lasciata alle spalle la tradizionale società rurale e le sue lingue (i dialetti) - e la tensione verso una medietas linguistica e stilistica, definita da Enrico Testa 'stile semplice', ossia l'orientamento verso 'la semplicità espressiva, intesa come adozione di una lingua narrativa media e per quanto possibile uniforme'. ${ }^{2}$ Sull'onda dei loro successi, alla metà degli anni Novanta diversi critici 
e linguisti si affrettarono a dichiarare 'decisamente finita (col trionfo nella società della lingua media e comune) la stagione in cui i narratori potevano affidare messaggi alle varietà geografiche e sociali del repertorio (con le loro immediate connotazioni geografiche e culturali)' ${ }^{3}$ Gli sviluppi successivi smentiscono però la drasticità di questa conclusione. Oggi appare evidente che la prosa plurilingue e radicata nel territorio, presente sin dalle origini nella nostra tradizione letteraria, non si è affatto esaurita. Lo dimostrano sia autori che hanno seguito fino agli anni Duemila percorsi letterari iniziati nei decenni precedenti, come Luigi Meneghello o Vincenzo Consolo, sia le nuove voci che si sono affacciate alla ribalta letteraria negli anni Ottanta-Novanta. Tra queste, si distinguono scrittori per cui il dialetto e l'italiano regionale concorrono con altri elementi alla resa di una patina di oralità gergale ${ }^{4} \mathrm{e}$ altri che utilizzano l'alterità linguistica per ragioni storico-antropologiche, in primis la difesa delle peculiarità locali contro le tendenze omologanti della contemporaneità. Consolo definisce questi ultimi 'scrittori verticali':

Quelli verticali siamo noi, i napoletani, i sudamericani, connotati da una realtà troppo forte per non affondare la scrittura in quelle radici. Maggiore l'infelicità sociale di una terra, più i suoi scrittori sono 'verticali' per il bisogno di spiegare il proprio dolore, di capirne il perché. ${ }^{5}$

Le opere dei giovani 'scrittori verticali' sono inizialmente trascurate dalla critica, anche perché talora proposte da piccole case editrici, meno visibili ma allo stesso tempo più disposte ad andare controcorrente rispetto alle regole del grande mercato, e quindi in grado di anticipare fenomeni di rinnovamento di lungo periodo. Il loro ruolo comincia a essere rivalutato solo di recente, in specie dopo l'esplodere di un fenomeno di massa quale quello della crime fiction regionale, spesso mistilingue; su tutti, si veda il successo del siciliano Andrea Camilleri, che, unendo un idioletto italo-siciliano a strutture narrative di facile leggibilità, ha messo in crisi l'associazione invalsa tra plurilinguismo, sperimentalismo ed elitismo.

A uno sguardo retrospettivo, dunque, meno schiacciato sugli eventi, il quadro degli anni Ottanta-Novanta appare più vario di quanto la critica coeva abbia messo in luce. Il I993, per esempio, è non solo l'anno del successo di vendite di Oceano mare di Alessandro Baricco, ${ }^{6}$ ma anche la data dell'inizio, in sordina, di tre percorsi di scrittura all'insegna del plurilinguismo e del radicamento geoetnografico. Giuseppe Ferrandino pubblica Pericle il Nero, ${ }^{7}$ giallo pulp partenopeo in cui la violenza linguistica è in sintonia con l'ambientazione nelle degradate periferie napoletane, mentre la lombarda Laura Pariani e la siciliana Silvana Grasso si presentano ai lettori con Di corno o d'oro ${ }^{8}$ e Nebbie di Ddraunnàra, ${ }^{9}$ raccolte di racconti rimaste a lungo nell'ombra nonostante le segnalazioni a premi letterari e da parte di recensori influenti. ${ }^{\text {Io }}$ Nella tradizione della narrativa plurilingue italiana, Pariani e Grasso si distinguono per la fusione di alterità linguistica e alterità di genere: esse raccontano infatti storie al femminile, strettamente legate ai rispettivi paesi di provenienza, in idioletti di base italiana che accolgono dialetti e apporti altri, da lingue classiche o moderne, in direzione soggettiva ed espressionista per la Grasso, socio-antropologica per la Pariani. Stante la scarsità di studi di riferimento in questo ambito, e sulla Pariani in particolare, ${ }^{\text {II }}$ le pagine che seguono si focalizzeranno soltanto sulla scrittrice 
lombarda, per la quale il plurilinguismo è parte di una più ampia poetica della marginalità — di genere, geografica, sociale, generazionale — , che diviene punto di vista privilegiato per uno sguardo sul mondo. Il concetto di margine sarà analizzato in quanto centro irradiatore della sua narrazione, e individuato nella preferenza per gli spazi periferici e sospesi, per le voci e le vite improntate all'irregolarità, per le strutture narrative complesse. Le radici di tali scelte di stile e contenuto saranno rintracciate in fenomeni socioculturali quali la contestazione antiautoritaria dei movimenti degli studenti e delle donne e la controcultura degli anni Settanta, i cui stimoli culturali lievitano nel corso dei decenni. ${ }^{\mathrm{I}} \mathrm{Nel}$ privilegiare l'affondo critico su una sola scrittrice si seguono le linee guida poste sin dagli anni Sessanta da Maria Corti, che, nel dibattito sulle scritture compromesse con il dialetto, invitava a evitare le generalizzazioni e a 'sostituire alla polemica un lavoro di discriminazione, di distinzione fra le diverse funzioni, autentiche o no, che il dialetto ha attuato di recente nella espressione artistica, a lato della via maestra nazionale, e soprattutto un'indagine delle deformazioni che gli scrittori più o meno volutamente apportano alla materia dialettale'. ${ }^{\text {I3 }}$

\section{IN-BETWEEN: I LUOGHI E LE LINGUE DELLA NARRAZIONE}

Dall'esordio nel I993 a oggi Laura Pariani si è messa alla prova nell'arte del narrare in diverse forme: i romanzi e i racconti, preponderanti, sono affiancati da scritti per il teatro e per il cinema, traduzioni e collaborazioni con la stampa periodica. ${ }^{\mathrm{I} 4}$ Similmente a molte scritture al femminile, anche il suo raccontare è legato alle esperienze di vita; un autobiografismo non esplicito o intimista, bensì trasfigurazione di spunti della memoria personale o familiare in racconto collettivo di un ambiente e di un'epoca. Lo slogan femminista 'il personale è politico' si fa modello per un'azione sul mondo attraverso la letteratura, e il racconto, a partire dal conosciuto, sfocia nella denuncia dell'oppressione delle donne, dei bambini, dei deboli, di tutte le forme di emarginazione, povertà e ingiustizia sociale.

Le ambientazioni delle sue storie oscillano tra i paesi dell'infanzia nella valle del Ticino (Busto Arsizio e Magnago) e l'Argentina dell'emigrazione familiare (tra Buenos Aires e la Patagonia). Fino al romanzo La Signora dei porci (r999) predominano il lato lombardo e la rappresentazione di un'alterità geografica interna ai confini italiani; dal 2000, dopo il secondo viaggio in Argentina della scrittrice, ${ }^{15}$ la narrazione prima vira con decisione sul versante sudamericano, poi alterna tra i due poli. ${ }^{16}$ In realtà, già il racconto d'esordio, 'Di corno o d'oro', si apre nel I890 a 'Tilcara [...] alla fine dell'altopiano, sulla strada che va dall'Argentina alla Bolivia', ${ }^{17}$ paesino dove trova la morte l'emigrato Carlén, e si chiude nelle campagne lombarde, alla 'Corte di Ursi' nel I 855 , cronotopo della nascita malfatata del protagonista. La costanza dei riferimenti alla provincia italiana e argentina mette in evidenza come queste costituiscano ben più che una quinta scenografica. La scrittrice ne ricrea gli ambienti con attenzione lenticolare, disegnando dei tableaux vivants sulle condizioni degli 'ultimi della terra' ( $\mathrm{La}$ Signora dei porci, p. 202), in diversi momenti storici: nel presente, o più spesso in un passato vicino, tra il secondo Ottocento e il Novecento, con punte cinquecentesche ne La spada e la luna e La Signora dei porci. Più indagate sono le zone rurali, nel nord Italia o nel finis mundi della Patagonia, mentre la dimensione urbana è spesso un moto a luogo o uno spazio di attraversamento. ${ }^{18}$ 
Anche nelle opere ambientate nelle grandi città, il punto di osservazione è nei margini topografici e sociali, dai conventillos della periferia meridionale di Buenos Aires (la Boca, San Telmo, San Cristóbal, quartieri di estrema povertà in cui vivono gli emigrati italiani di Dio non ama $i$ bambini e Quando Dio ballava il tango), alla Milano del I969 percorsa dal barbone Dante, il cui sguardo dal basso guida la narrazione in Milano è una selva oscura. La dislocazione geografica è funzionale alla creazione di una tensione costante tra centro e periferia, alto e basso, Storia e storie.

La geografia letteraria della Pariani privilegia ciò che è ai margini dello spazio sociale e nazionale - per difetto (le micropatrie locali) o per eccesso (il transazionale) - e ferma l'attenzione sulle aree di contatto e frizione fra le diverse dimensioni. L'esperienza migratoria, in specie, in quanto in-between, condizione di attraversamento e luogo sospeso, provoca la messa in discussione delle identità individuali e collettive, che devono essere rinegoziate a ogni passo. A farsene simbolo sono soprattutto gli emigrati stagionali, golondrinas ('rondini') che fanno la spola tra vecchio e nuovo mondo, e le loro famiglie. Tra questi il Togn, la cui migrazione in Argentina dà inizio all'epopea di Quando Dio ballava il tango:

Due pianeti paralleli, L'Italia e l'Argentina, in cui per un po' si può anche pensare di riuscire a barcamenarsi: bilanciandosi in quella zona intermedia che si chiama equivoco, ambiguëdad. Ma guai se le orbite dei due mondi si incrociassero: sarebbe la catastrofe. (Quando Dio ballava il tango, p. 23)

Per il Togn l'incrocio di orbite nazionali ritenute parallele sovverte le regole conosciute e costituisce una catastrofe da evitare; difatti, non riuscendo a superare la nostalgia dell'altrove insita nella condizione di pendolare transoceanico, finisce per tagliare i legami con l'Italia e recuperare un'esistenza stanziale, con una nuova famiglia, in Argentina. La parola del personaggio è però, bachtinianamente, bivoca, ${ }^{\text {I9 }}$ ossia condensa in sé anche il punto di vista opposto dell'autrice, per la quale proprio le condizioni di equivoco e 'ambiguëdad' identificano il margine di dolore e spaesamento da cui vivificare, con la complessità delle storie individuali, le gabbie identitarie. Con una forte opposizione generazionale e di genere, la logica binaria del capostipite è ribaltata dalla discendente Corazón, che in chiusura del romanzo, a cento anni di distanza, accetta come condizioni esistenziali lo sradicamento e il marchio della diversità, l'appartenenza plurima e lo sfocare dei confini identitari:

[...] adesso che ha sperimentato sulla propria pelle cosa si prova a vivere in una terra dove non si è nati, parlando un'altra lingua con un accento mai perfetto, quasi un marchio di diversità: come se, invece di appartenere a due paesi, non si appartenesse a nessuno. Confondendo uno y otro. (Quando Dio ballava il tango, p. 29I)

Ancor prima che al concetto di in-between elaborato nell'ambito dei postcolonial studies, ${ }^{20}$ lo stato di sospensione e crisi delle rigidità identitarie dei personaggi della Pariani, e con esso la tensione verso una ridefinizione non binaria delle identità individuali e collettive, possono essere messi proficuamente in rapporto con l'in-between arendtiano ripreso da Adriana Cavarero, in quanto 'ciò che mette 
in relazione e separa gli uomini allo stesso tempo, rivelando la loro condizione plurale', ovvero 'relazione ontologica iscritta nella pluralità dei singolari' ${ }^{2 I}$

I pensieri di Corazón rimarcano inoltre il nesso tra lingua/e e identità che, in coerenza con l'impostazione ex-centrica, giustifica l'idioletto della Pariani: un italiano letterario, raffinato e sperimentale, arricchito dai dialetti dell'infanzia — il bustocco di famiglia, il lombardo occidentale, le parlate del basso Ticino, fino alle valli alto-piemontesi ne La valle delle donne lupo 22 - e dallo spagnolo nella declinazione spuria e approssimativa dei tanos, gli emigrati italiani in Argentina. In Milano è una selva oscura, le fonti letterarie del dialetto sono evidenti e dichiarate: il meneghino è ricostruito senza intenti filologici ma con scrupolo, sulla scorta della tradizione letteraria lombarda - le traduzioni dialettali dell'Inferno dantesco di Porta, la narrativa (da Manzoni a Dossi e Gadda), la poesia (Tessa), il teatro (Fo) - e con l'ausilio del Dizionario milanese-italiano ottocentesco di Cletto Arrighi. ${ }^{23}$ In genere, però, più che la letteratura colta e la consultazione dei dizionari, a irrorare il testo è il dialetto parlato, sedimentato nella memoria acustica, recupero delle voci dell'infanzia paesana, in specie di donne, anziani, bambini; non è un caso che la lettura ad alta voce, cui la scrittrice si dedica nei tour di presentazione dei libri, siano di aiuto nel processo di comprensione dei testi, perché riportano la parola in dialetto alla dimensione originaria di oralità, liberandola dalla prigione dell'inusuale, e talvolta ostica, resa grafica. Gli intertesti sono i racconti di famiglia, o quelli raccolti nel corso di interviste, e materiali demotici (quali canzoni, conte, filastrocche, proverbi, credenze, modi di dire) che fondano la sapienza popolare, tramano i testi della scrittrice. ${ }^{24}$ Anche sul fronte spagnolo la fascinazione è per le parole tramandate a voce, e quindi le canzoni, i proverbi, le parlate dei tanos analfabeti e dialettofoni, e per l'interazione della loro babele di dialetti con lo spagnolo, da cui si originano i gerghi del cocoliche (l'italiano-spagnolo degli immigrati italiani a Buenos Aires) e del lunfardo (argot della malavita di Buenos Aires). ${ }^{25}$

Nonostante la mescidazione linguistica sia il marchio di fabbrica della scrittura della Pariani, ${ }^{26}$ a tutt'oggi mancano analisi linguistiche, anche solo parziali, del suo idioletto; sono quindi ancora da indagare la natura dei prestiti, le modalità specifiche della loro inserzione all'interno dell'italiano letterario, le fonti. Anche una lettura non specialistica, però, può evidenziare come, a fronte di un numero limitato di citazioni pure (dialettali o straniere), la scrittrice dedichi attenzione all'incontro tra sistemi linguistici, agli italiani regionali e popolari e ai creoli internazionali, pratica favorita dal ricorso frequente al soliloquio dei personaggi e al discorso indiretto libero. Così, per esempio, la descrizione del risveglio di Ipólita, una delle protagoniste de La Signora dei porci, nella Magnágu cinquecentesca, trascolora nel flusso di coscienza del personaggio, alternando italiano e lombardo:

La moglie dul Giuachén si risvegliò per prima. Le sembrava che il sonno stesse per tornare, ma di colpo le venne alla mente l'Antóni. Scacciò con rabbia il pensiero di quel bagulûn dul lüstar ${ }^{27}$ e chiuse con forza gli occhi, ma davanti a lei, invece dello scuro fluire dul sogn, comparivano gli ögi dul Magatèl. O fiurutti dul signùr mortu, livé-sü che ul sô l'è vóltu. Ora di valzarsi, s'ciàu. (La Signora dei porci, p. I I4)

O ancora, in contesto sudamericano: 
Quando il Carlén porta il discorso sulle lettere, il Miliu si mette a parlar d'altro, come se avesse le fragùglie sotto il culo; e la voce del Carlén si fa rabbiosa e acida, más pronto que la leche. Oltre alle cibarie di vario tipo il carro trasporta anche balle di stoffa, bottoni, corda, bombàso per scarpe, aghi [...] una vera bisa-bòsa. ('Di corno o d'oro', p. 24)

Qui l'italiano della narrazione subisce la doppia interferenza del substrato dialettale e del superstrato spagnolo: nel discorso indiretto libero, la voce italiana della narratrice si incrocia con il punto di vista e le parole del Carlén, incorporando termini lombardi ('bombàso', ['cotone'], 'bisa-bòsa', ['confusione', 'guazzabuglio']) e modi di dire sia in italiano regionale ('avere le fragùglie [briciole] sotto il culo', ['avere fastidio', 'innervosirsi']), ${ }^{28}$ sia argentini ('más pronto que la leche', ['molto velocemente']), resa linguistica di un'identità stratificata e di un'appartenenza plurima. Stilisticamente, il mistilinguismo crea vuoti semantici che il lettore è chiamato a riempire con uno sforzo ermeneutico suppletivo (di ricerca o di immaginazione), potenzia l'ambiguità che è precipua della parola poetica e ne moltiplica i significati. Ideologicamente, scardinando la presunta monoliticità della lingua nazionale, pone degli interrogativi sulla definizione dell'identità (individuale e collettiva), il cui incontro con l'Altro da sé non avviene in termini di giustapposizione.

\section{VOCI E ARCHITETTURE TESTUALI}

L'orchestrazione linguistica della Pariani si inquadra bene nel discorso elaborato da Bachtin sull'impossibile neutralità della parola letteraria e sul concetto di plurilinguismo, ossia sull'opposizione tra la lingua unitaria, risultato di un processo di unificazione e centralizzazione politico e culturale, e le forze centrifughe che la frammentano nei socioletti e negli idioletti. ${ }^{29}$ Risulta utile anche il ricorso alla definizione bachtiniana di 'romanzo polifonico', spazio testuale in cui dialogano e si scontrano le voci di narratore e personaggi, che sono 'non soltanto oggetti della parola dell'autore, ma anche soggetti della propria parola immediatamente significante': ogni voce è un ideologema, ossia una parola che esprime un'intenzionalità e un punto di vista sul mondo. ${ }^{30}$

Le teorie narratologiche di Bachtin si sviluppano a partire dall'analisi del romanzo ottocentesco con narratore esterno (Dostoevskij), ma tali procedimenti di costruzione del testo sono spinti alle estreme conseguenze nel romanzo postmoderno, d'impronta metanarrativa e metalinguistica, che predilige l'alternanza delle voci narranti e dei punti di vista, la scomposizione e l'incastro dei piani narrativi, e che, oltre a procedere per ellissi, scarti temporali e frammenti, problematizza la figura del narratore. Di questa temperie letteraria partecipa la Pariani, per la quale la forma romanzo ha perso ogni ambizione totalizzante ed è articolata, perlopiù, in una concatenazione di storie brevi, racconti in sé compiuti e affidati a diversi narratori, inseriti in una cornice contemporanea dove spesso compare una proiezione testuale dell'autrice. La voce narrante non è anonima e oggettiva, ma soggettiva, personalizzata e personificata; ne viene ribadita l'appartenenza di genere (è una narratrice), e il suo ideologema è avvicinato a quello dei personaggi e dunque relativizzato. Da interprete della Storia, la narratrice diviene raccoglitrice e trascrittrice delle storie altrui. Il suo ruolo è 
narrativizzato all'interno dei testi, in cui compare spesso come erede di una genealogia femminile non scritta, che le impone il recupero della memoria grazie all'arte della parola e del racconto. La 'Scrittrice' che nelle 'Notti' della scrittura conversa nella sua camera con il 'Mietitore' (la morte), in una corte lombarda, alla ricerca di 'una storia con una donna seduta a filare in un cantuccio' (La Signora dei porci, p. 6), si pone il compito di salvare dall'oblio le vite della vecchia Pulónia, di sua figlia Ipólita e della nipote Sanguèta: 'ste donne di Magnágu, ché nessun ricordo farà mai eco al loro essere falciate' (p. 253). ${ }^{3 \mathrm{I}}$ Laura - l'etnografa, la 'sciura milanese' de La valle delle donne lupo - ascolta e registra su nastro la storia della vecchia Fenísia - la lupa, 'balenga', 'stròliga', 'strÍla' —, che a sua volta è investita del ruolo di portavoce delle donne delle generazioni precedenti: '[q]uando torna a casa, [...] sembra di avvertire sulle spalle lo sguardo di una schiera grigia e spettrale: quelle che nessuno piange, quelle che nessuno vuol ricordare, quelle che non hanno neppure una lapide col nome' (p. I67). Il ruolo della narratrice in quanto anello finale della trasmissione delle storie femminili si definisce con ancora più forza in Quando Dio ballava il tango, controstoria dal punto di vista delle donne di cento anni di migrazioni italiane in Argentina. Il romanzo è organizzato come sequenza di sedici ritratti, dalla figlia del capostipite Togn, Venturina Maina (I892-I98I), rimasta in Italia, fino a Corazón Bellati (1952), nata in Argentina e fuggita in Italia dopo l'avvento della dittatura e la scomparsa del compagno, desaparecido. Le cornici qui sono due. La prima, posta in apertura, con l'arrivo di Corazón presso Venturina alla Cascina Malpensata nel I978, ricongiunge i lati sudamericano e italiano della famiglia e avvia ricordi e racconti:

Perché mi viene così facile parlare a questa ragazza mai vista? — riflette Venturina Non so, ma mi rendo conto che è a me che tocca a questo punto contare storie, rivelare i segreti ingannatori; le chiavi di quel mondo dove, con la partenza del mepà, tutto ebbe inizio. E, nel contempo, capisco che dopo morirò, che il mio compito sulla terra sarà esaurito, ma è un sapere che mi dà sollievo. (Venturina, in Quando Dio ballava il tango, p. 22)

Nell'ultimo capitolo il lettore scopre che la prima cornice è incastonata in una seconda più tarda, contemporanea al tempo della scrittura (200I) e locata a Buenos Aires; è qui che Corazón, la quale 'ha preso l'abitudine di parlare ai suoi morti, nella penombra del videoregistratore' (p. 287), passa le notti a recuperare dai nastri raccolti il senso di una genealogia sparsa tra i due continenti, prima che le memorie individuali scompaiano: 'Tutto è accaduto tanto tempo fa; quel che la vecchia sa e ricorda cesserà di esistere tra qualche mese o anno, con la sua morte' (p. 299). Come la 'Scrittrice' e la 'sciura Laura', Corazón è l'erede delle donne che hanno preservato la storia familiare femminile nella forma del racconto orale, secondo un imperativo etico reso esplicito da Venturina in Italia e da Catterina in Argentina. È infatti la bisavuela Catte, come farà poi Venturina, a passare alla nipote il testimone di uno dei bandoli della memoria femminile familiare, di cui lei stessa si è fatta carico:

A metterle in bocca ogni frase era un'intera stirpe di donne, che avevano fatto un lungo apprendistato con la morte; impossibile farle tacere. [...] 
Davanti agli occhi le sfila un corteo di donne morte. [...] tutte, con l'espressione del volto, sembrano esigere dalla Catterina che dia testimonianza di come vissero e soffrirono: basterà un nome, una data, un misero aneddoto da raccontare per liberarle dal pericolo di scomparire nell'oblìo. Lei può farlo, ché è sopravvissuta, vuoi per caso, vuoi perché altre morirono al suo posto. (Quando Dio ballava il tango, pp. 79 e 83 )

Corazón, Laura, e la 'Scrittrice' rappresentano un punto di svolta nella catena delle memorie femminili perché, vivendo nella contemporaneità e avendo avuto accesso a un percorso formativo paritario a quello degli uomini, sono in grado di traghettare le storie delle donne dalla dimensione orale e familiare a quella scritta e letteraria, favorendone dunque l'ingresso nella Storia. L'arte del 'contar storie' è sempre presentata nell'opera della Pariani come una delle poche ricchezze a disposizione delle donne, un sollievo al dolore di fondo dell'esistenza, per quanto temporaneo e circoscritto. ${ }^{32} \mathrm{La}$ trasposizione delle storie nella pagina letteraria, però, compie un passo ulteriore: passa il testimone della memoria a un numero potenzialmente infinito di lettori, facendo uscire le donne dall'oscurità e dall'oblio. È evidente nei testi la tematizzazione di uno dei cardini del progetto letterario della Pariani, che nella scrittura non rinuncia a ricreare un ambito di oralità, sia nell'organizzazione delle voci del testo, con ampio spazio ai personaggi e alle loro digressioni, sia nella citazione di testi orali della sapienza popolare, sia nel racchiudere le storie in interni casalinghi, dove avvengono le conversazioni intergenerazionali, spesso al femminile. ${ }^{33}$ Contro la vulgata che legge nel postmoderno italiano l'età del disimpegno, per la Pariani la letteratura rimane una missione a impronta etica, impegno dei vivi verso i morti, atto che spiega il presente tramite il passato, arma di riscatto proiettata nel futuro:

ché la letteratura può anche essere gesto di libertà, di salvezza, perfino di redenzione; e nelle pagine dei libri le sorti del passato possono venir buttate all'aria: per cui da una parte i principi padri e i fratelli despoti, un tempo vincenti, ora sono schiacciati per l'eternità dal nostro disprezzo; e dall'altra le donne, che furono forzate e sconfitte, ancora possono rivolgerci uno sguardo di sogno. ${ }^{34}$

\section{RACCONTARE LA SUBALTERNITÀ ALL'INTERNO DELLA LINGUA EGEMONE}

Claudia Nocentini, nel presentare l'opera della Pariani, richiama due pilastri del secondo Novecento: Italo Calvino, secondo il quale uno degli 'usi politici giusti' della letteratura è nel dare voce a quelli che non ne hanno, e Umberto Eco, che invita a misurare la sfida etica dello scrittore nelle strutture letterarie, più che nella mera scelta dell'oggetto narrativo (la referenzialità delle storie). ${ }^{35}$ Nell'opera della scrittrice, difatti, la volontà di soffermarsi sui destini delle vite marginali è tutt'altro che un'operazione di realismo ingenuo. Le tendenze riflessive, metalinguistiche e metanarrative, la costante tematizzazione dell'atto del raccontare e la presenza della scrittrice ai margini delle storie come figura testuale, da un lato ribadiscono che tanto la voce narrante quanto i personaggi sono individualità contingenti ma separate, non riducibili l'una nell'altra, dall'altro rimarcano la distanza tra il lettore e la storia. Inoltre, con la scelta di un 
plurilinguismo che non prevede l'uso di corsivi o virgolette, né traduzioni in nota né glosse esplicative, la scrittrice erige tra il lettore e il mondo narrato una barriera, per quanto sormontabile, con maggiore o minore difficoltà, grazie alla radice latina che accomuna italiano, dialetto e spagnolo. ${ }^{36}$ Anche all'interno delle storie, la comunicazione tra i personaggi è resa difficile dalla barriera linguistica, come mostrano due passi tratti dalle sezioni di cornice di Quando Dio ballava il tango e La valle delle donne lupo:

"Non so se parlo chiaro. Intende? Voglio dire: intendi quello che dico? Ché io parlo un po' dialetto, ne"”. (Venturina a Corazón, in Quando Dio ballava il tango, p. 20)

È forse perché non parla pulito? Perché dalla bocca le esce sto purpurrí di dialetto e italiano? [...] La sciura deve perdonarla, il dialetto lei l'ha succhiato col latte. Le parole sono come le braccia e le gambe di quello che una persona è. Eccosí la Fenísia è la sò lingua. (Fenísia a Laura, in La valle delle donne lupo, p. Iо4)

L'ammissione della difficoltà comunicativa, nel ribadire l'alterità linguistica, culturale e sociale del soggetto della storia narrata rispetto alla voce che la narra, richiama il paradosso della delega indicato da Gajatri Spivak come limite insito nelle retoriche dei postcolonial e subaltern studies, ossia la foucaultiana 'violenza epistemica' operata su un gruppo subalterno (in questo caso le donne 'invisibili', tra valle del Ticino e il Sudamerica) dallo stesso 'benintenzionato' intellettuale occidentale che si fa portatore delle ragioni del gruppo (la scrittrice). ${ }^{37}$ In quest'ottica, tradurre in italiano letterario le storie dialettali e orali delle protagoniste, ossia trasferire nella lingua scritta ed entro le categorie della cultura egemone la marginalità delle loro vite subalterne, rischia di costituire l'ennesima forma di colonizzazione dell'immaginario e dell'espressione di chi storicamente non ha avuto voce. La Spivak insiste provocatoriamente sulla non-trasparenza e fondamentale inconoscibilità del soggetto rappresentato, fino alla lapidaria conclusione che il subalterno non può parlare se non come 'informante nativo' dell'anticolonialista intellettuale metropolitano, di cui sostiene, talvolta senza averne consapevolezza, il progetto neocoloniale..$^{3}$ D'altra parte, la Spivak, teorica del decostruzionismo e intellettuale post-gramsciana e femminista, non predica la rinuncia alla rappresentazione (p. 285). Piuttosto, indica la strada da seguire nell'assunzione di responsabilità da parte dell'intellettuale, che deve avere consapevolezza della problematicità con cui si relaziona alla questione della coscienza e della voce del soggetto subalterno, e doppiamente nel caso delle donne, perché '[w]ithin the effaced itinerary of the subaltern subject the track of sexual difference is doubly effaced' (p. 274). Per fare ciò, l'intellettuale deve riflettere su se stesso/a e sulla propria identità personale e sociale, sulla sua posizione rispetto al soggetto subalterno, in termini etnici, sociali, di classe, economici, di genere ('posizionalità'): 'To confront this group is not only to represent (vertreten) them [...], but also to learn to represent (darstellen) ourselves' (p. 276).

Non dissimili, come si è visto, sono i principi ideologici che ispirano la Pariani nella scelta di luoghi marginali e di protagonisti subalterni, nella distanza tra chi conduce le fila della narrazione e l'alterità irriducibile dei soggetti raccontati, nella narrativizzazione della propria posizionalità: elementi che indicano come la scrittrice non rinunci all'impegno di dare voce a quanti sono ridotti al silenzio 
dalla Storia, evitando però di rendere l'Altro trasparente e pienamente conoscibile, e accompagnando l'indagine sull'Altro con l'autoriflessività. ${ }^{39}$

Spivak prende spunto dallo sguardo accademico postcoloniale sulla donna indiana, mentre la Pariani si focalizza su soggetti subalterni (principalmente femminili) interni alla dimensione nazionale o in precario equilibrio transnazionale, ma entrambe, pur a distanza geografica e culturale, si sono nutrite del pensiero gramsciano e femminista. In particolare, per la Pariani, studentessa di filosofia alla Statale, le radici sono nella controcultura milanese della prima metà degli anni Settanta, tra impegno politico nella sinistra extraparlamentare e militanza nei collettivi di donne, dal grande centro aggregatore di via Cherubini al più politicizzato 'Lotta Femminista', esperienze fondanti di cui la scrittura letteraria è una continuazione creativa. ${ }^{40}$ Anche nelle articolazioni narrative, le ricorrenti sezioni di testo che presentano la creazione e trasmissione delle storie come risultato di un dialog $\mathrm{O}^{4 \mathrm{I}}$ si possono leggere come rielaborazione, oltre che dello storytelling familiare dell'infanzia, della pratica dell'autocoscienza dei collettivi femministi, in cui la condivisione delle storie di vita individuali è funzionale alla comprensione e decostruzione dei meccanismi di funzionamento del sistema patriarcale. ${ }^{42}$ All'interno dei suoi romanzi, inoltre, si delinea un contesto di definizione del soggetto comunicante e della sua relazione con l'Altro all'insegna non dell'omologazione razionale bensì dell'unicità della voce parlante, su cui, ancora una volta, sono illuminanti gli schemi di base arendtiana proposti dalla Cavarero:

Lungi dal sottolineare la valenza normativa, la razionalità, l'universalità e l'orientamento all'intesa del linguaggio, Arendt insiste su un 'concetto del parlare' dove ciò che viene in primo piano è l'atto relazionale e contestuale del comunicarsi, gli uni agli altri, degli unici. [...] Crucialmente, coloro che in tal modo si comunicano, non sono gli individui. Sono degli esseri unici in carne e ossa che, al contrario dell'individuo astratto e universale, hanno un volto, un nome, una storia. Irripetibili e diversi da ogni altro, essi comunicano su una scena condivisa l'unicità della loro identità personale. Comunicano, reciprocamente, chi sono. ${ }^{43}$

Le opere di narrativa, peraltro, riprendono senza discontinuità di rilievo i temi affrontati nelle primissime prove di scrittura, i fumetti pubblicati nel I975-76, Perché non i fiori e La fata rovesciata, incentrati sulla denuncia dell'oppressione della donna nella società contemporanea. ${ }^{44}$ I fumetti non sono ancora sperimentali sotto il profilo linguistico, ma il linguaggio è chiaramente indicato come l'ennesima gabbia sociale che imprigiona il soggetto subalterno. ${ }^{45} \mathrm{Ed}$ è secondo schemi di classe e di genere che si costruisce, per esempio, il plurilinguismo de La Signora dei porci che, nel quadro della violenza istituzionalizzata del processo, oppone all'italiano e al latino degli inquisitori il dialetto rustico delle donne. Né il notario del Sant'Uffizio né il suo vicario sono disposti ad accettare il monolinguismo dialettale della popolana Pulónia, la 'stròliga', la 'stría', perché le regole della comunicazione sono dettate da chi detiene il potere. La parola qui non è veicolo di incontro ma strumento di esclusione e oppressione:

Che volevano ancora questi due uomini da lei? Difficile capire il vicario: parlava in cìmberli, con un tale accento furasté, quasi incomprensibile, per cui lei che, da cieca, 
conosceva gli altri solo per quel che usciva dalle loro labbra, per tutta la durata del processo s'era sentita spaesata. (La Signora dei porci, p. 8$)^{46}$

L'oppressione linguistica, finalizzata a tenere sotto silenzio i subalterni, ritorna nelle memorie della scuola dialettofoba fascista della donna-lupo Fenísia, che ne connette esplicitamente $\mathrm{i}$ meccanismi a quelli della violenza coloniale in Sudamerica:

[...] quand'era piccola, il maestro sgridava in faccia ai bocètti: 'Voi non avete lingua!'

[...] Ché a quei tempi era obbligatorio parlare italiano: tutti a ripetere a alta voce parole stravaganti come 'babbo', 'glauco', 'aiuola' come pompando il fiato. Ché la prima mira del maestro Anselmo era di metter sulle labbra dei bocètti quelle strane espressioni: non per far paragoni, neh, ma preciso a uno di quei missionari che insegnano ai mammelucchi a far il segno della croce; eppoi magari con una mano gli spruzzan l'acquasanta in fronte per battezzarli e con quell'altra gli tengono la spada puntata alla gola. Ma loro, i bocètti del paese, mica si arrendevano. See. Epperò maestro è maestro. E chi sta in cattedra ha sempre il coltello dalla parte del manico. (La valle, pp. $\left.\mathrm{IO}_{4}-05\right)^{47}$

Erede delle 'stríe' e delle donne-lupo, la scrittrice accetta la lingua egemonica della tradizione letteraria per forzarla dall'interno con i residui linguistici della marginalità dei personaggi, ribaltando così i rapporti di forza e costringendo il lettore allo sforzo della comprensione linguistica, primo segno di una volontà di ascolto verso la voce degli esclusi.

Anche l'attenzione per la riscrittura della storia a minore, secondo il punto di vista dei vinti, riporta alla formazione giovanile della scrittrice. Al suo modus narrandi, attento ai dettagli della vita materiale nelle campagne, con il lavoro nei campi e i piccoli commerci per gli uomini, le mansioni domestiche per le donne, ${ }^{48}$ non è estranea la lezione della microstoria, della storia locale e della storia orale che si sviluppano in Italia dalla fine degli anni Sessanta, sulle cui finalità riporto la precisazione di Carlo Ginzburg:

la microstoria non si occupa di piccola storia, si occupa di storia in maniera analitica, con uno sguardo ravvicinato. [...] È lo sguardo che conta; le domande che facciamo. Io penso che la microstoria ponga sempre, in maniera esplicita o implicita, un problema di generalizzazione. Si parte da un caso circoscritto (tutti i casi, grandi o piccoli, lo sono) per capire qualcosa di più generale. Magari si tratta di un caso anomalo: ma ogni anomalia contiene in sé la norma. Non è vero l'inverso. ${ }^{49}$

Queste forme di ricerca, con i temi che sviluppano e le preoccupazioni metodologiche su cui si fondano, appaiono come intertesti imprescindibili della narrazione della Pariani. La tendenza all'ibridazione di racconto, saggistica e autobiografia richiama gli scritti di Luisa Passerini, ${ }^{\circ}$ mentre il processo alle 'stríe' lombarde de La Signora dei porci è quasi una riscrittura al femminile di quello per eresia contro il mugnaio friulano Menocchio, ricostruito da Carlo Ginzburg sulla base di micro-letture di documenti dell'Inquisizione ne Il formaggio e i vermi.$^{5 \mathrm{I}} \mathrm{Se}$ l'articolarsi del racconto rimanda alla pratica di ricerca dell'etnografo che conduce le interviste sul campo o dello storico negli archivi per riportare alla luce storie 
dimenticate, la stessa scrittrice ricorda come l'urgenza della scrittura si sia presentata, alla fine degli anni Ottanta, a latere di una ricerca storica a quattro mani, poi abbandonata:

Quanto alla Storia, quella dei libri di storia, non mi interessa molto, preferisco raccontare le storie marginali. Quando ho iniziato a scrivere Di corno o d'oro avevo in mente di scrivere con un mio amico un libro di storia sul movimento contadino a partire dall'unità d'Italia, e mi ero documentata in proposito. Mentre consultavamo i documenti, venivano alla luce cose bizzarre, definite 'robaccia' dal mio amico, ma da cui invece io ero attratta. I protagonisti di queste storie non avevano niente a che fare con la Storia con la esse maiuscola, quella fatta dai grandi eventi. Lì mi sono resa conto che non dovevo scrivere un libro di storia, che a me insomma interessavano le storie possibili. ${ }^{52}$

\section{QUESTIONI DI GENERE E LINGUA NEL CANONE DELLA CONTEMPORANEITÀ}

L'adozione di un punto di vista marginale, la scelta del plurilinguismo e lo sguardo femminile fanno sì che la Pariani si inserisca con originalità nel canone della narrativa del terzo millennio. Da un lato, la scrittrice si è rapportata con scetticismo all'interpretazione secondo la quale la letteratura scritta da donne è una sovrapposizione tra l'identità di genere di chi narra, le tematiche trattate e l'impostazione ideologica e, problematizzando la definizione di un canone letterario al femminile, ha precisato che '[n] on sono le tematiche a fare un'autrice "femminista". Le tematiche strettamente legate alla condizione della donna non determinano niente di per sé, anzi a volte possono essere il segno dell'accettazione di un "ghetto"". ${ }^{53}$ Allo stesso tempo, ha fornito indicazioni su almeno due scrittrici di riferimento, Maria Bellonci, per la ricchezza e i dettagli dei quadri storici al femminile, ${ }^{54}$ e Armanda Guiducci, la cui figura ci riporta ancora una volta alla riflessione sulla condizione femminile degli anni Settanta. Il suo La donna non è gente (I977), menzionato in recenti conversazioni come intertesto de La valle delle donne lupo, ${ }^{55}$ è un saggio tanto influente all'epoca quanto poco conosciuto oggi: un'indagine basata su interviste a donne emarginate dalle battaglie femministe coeve, rintracciate nei luoghi di lavoro in diverse regioni italiane; anche qui, la militanza femminista appare come un lusso riservato a chi appartiene alla cultura egemone, secondo una linea di riflessione non distante da quella sviluppata poi dalla Spivak.

Più in generale, nel dibattito sul canone femminile l'opera della Pariani invita a un ripensamento delle linee di tendenza dell'ultimo ventennio e delle genealogie letterarie delle narratrici contemporanee. L'ultima parte della ricognizione sulle scrittrici italiane del Novecento proposta da Sharon Wood, dedicata agli anni successivi al miracolo economico (I964-94), è incentrata sulle figure di Dacia Maraini, Francesca Durante, Sandra Petrignani e Paola Capriolo, esponenti di un 'move in contemporary writing by women from femminism as a political movement to the current fragmentation of a costellation of writers who no longer align themselves to ideologies or party politics'. ${ }^{56}$ Nell'abbinare la questione del genere con quella della lingua e con la prospettiva di classe, la Pariani ritorna invece alla centralità dell'ideologia e del discorso politico nella creazione letteraria, 
dimostrando come il femminismo militante degli anni Settanta non si esaurisca necessariamente nella scrittura del riflusso degli anni Ottanta, ma ricompaia come una corrente carsica nei Novanta-Duemila. In particolare, nella Pariani emerge l'eredità di quante hanno inteso l'impegno nel movimento delle donne, prima che come sviluppo in chiave psicoanalitica di un pensiero della differenza femminile, come azione diretta e immediata sulla realtà per la rivendicazione dei diritti civili, una pratica che all'epoca univa alle sedute di autocoscienza il volontariato nei consultori di self-help o l'autogestione degli asili. ${ }^{57}$ In quest'ottica si capisce perché la focalizzazione sulle storie di donne sia, nell'opera della Pariani, distintiva ma mai unica. La marginalità di genere non può essere letta nell'ignoranza o disinteresse per altre forme sociali di esclusione: metonimicamente, la donna rappresenta tutti i senza voce, dai bambini ai poveri, ai vagabondi, ai folli. La sua poetica del dolore e della marginalità, piuttosto, si ricollega a scrittrici degli anni Sessanta come Elsa Morante e, soprattutto, Anna Maria Ortese: si pensi a un romanzo come L'Iguana (1966), in cui viene esplorata, con i toni del fantastico, l'alterità irriducibile della protagonista, donna subalterna, agli occhi delle élite coloniali locali (Ilario) e degli intellettuali occidentali (l'editore milanese, il conte)..$^{8}$

L'ultimo elemento da mettere in rilievo, in chiusura, è l'importanza delle questioni linguistiche per il posizionamento della Pariani nel canone della narrativa contemporanea. Come si è visto, il plurilinguismo italo-lombardospagnolo è un elemento fondante del suo progetto letterario, e non può essere scisso dal sostrato poetico e ideologico che lo motiva. Eppure, in traduzione, la Pariani è soprattutto scrittrice dell'emigrazione italiana in Argentina, ${ }^{59}$ e le selezioni della sua opera proposte dalla critica si focalizzano per lo più sui pochi scritti tendenti alla narrazione piana e al monolinguismo. Nella raccolta canonizzante dei Racconti italiani del Novecento, curata da Enzo Siciliano per la collana 'I Meridiani' della Mondadori, per esempio, compare con if racconto monolingue 'I nottambuli', ispirato alla pittura (il quadro omonimo di Edward Hopper) e al cinema (l'attore James Dean). ${ }^{60}$ Nell'antologia, il cappello introduttivo ribadisce inizialmente come '[p]er Laura Pariani va[da] segnalato il modo in cui la tradizione veristica lombarda, passata nelle mani del Gadda dell'Adalgisa, possa ancora trovare officianti e adepti', ma, dopo una serie di riferimenti a Gentile, Wittgenstein e Lacan, il curatore opta per un 'racconto disteso', in cui 'il suo stile si è decantato, rasserenato', quasi a suggerire l'equivalenza tra maturità letteraria e superamento della complessità plurilingue suggestione puntualmente smentita dalla produzione successiva. ${ }^{6 \mathrm{I}}$ Tale parzialità di approccio conferma il disagio verso il mistilinguismo comune a parte della critica contemporanea, un disagio connesso da un lato a una polemica contro Gianfranco Contini, dall'altro all'incapacità di svincolarsi dal nesso plurilinguismo-espressionismo istituito implicitamente nella sua 'linea-Gadda'. ${ }^{62} \mathrm{E}$ ciò nonostante, sin dai primi anni Novanta, Alfredo Stussi e Pier Vincenzo Mengaldo abbiano messo in guardia dall'operazione di restringimento del plurilinguismo alla sola 'tradizione della soggettività', che sacrifica le ragioni di poetica individuale di altri autori, schiacciandoli sul modello gaddiano. ${ }^{63}$ È opportuno domandarsi se verismo ed espressionismo siano ancora utilizzabili come macro-categorie entro le quali chiudere tutta la varietà delle scritture mistilingui: Verga e Gadda possono 
essere ancora punti di riferimento per gli scrittori contemporanei, ma secondo modalità da discernere caso per caso.

Nell'analizzare il plurilinguismo di Laura Pariani si evince bene la necessità di considerare l'interazione di molteplici fattori. La consolidata tradizione letteraria regionale lombarda, in prosa e in versi, scritta e orale, è presente ma filtrata dalle forme narrative e dai rovelli ideologici del postmoderno e si arricchisce inoltre degli stimoli derivanti da una storia familiare di emigrazione, dalla riflessione sulla differenza di genere, dall'interesse etno-antropologico per la marginalità, dalla difesa delle micropatrie e delle loro lingue contro le pressioni omologatrici della società contemporanea. Un approccio generazionale, sorretto da sistematiche analisi linguistico-stilistiche e narratologiche degli scrittori italiani plurilingui nati negli anni Cinquanta, ${ }^{64}$ per esempio, porterebbe nuovi elementi di riflessione sia in merito alle poetiche dei singoli, sia in relazione ai mutamenti della società italiana negli ultimi sessant'anni, dal regresso della dialettofonia per la spinta dell'accresciuto uso dell'italofonia (e il conseguente passaggio del dialetto 'da lingua della realtà a lingua della poesia'), ${ }^{65}$ ai processi di rinnovamento democratico, compresa la parità di genere, innescatisi sull'onda del Sessantotto lungo. ${ }^{66}$

E ormai urgente un rinnovamento delle categorie di inquadramento dell'irregolarità linguistica in letteratura che, senza ignorare gli elementi di continuità con la tradizione, sostanzi però l'interpretazione del dato stilistico con la dimensione ideologica, letta come risposta individuale agli stimoli socioculturali di un determinato momento storico.

\section{NoTE}

I Cfr. Stefano Tani, Il romanzo di ritorno. Dal romanzo medio degli anni Sessanta alla giovane narrativa degli anni Ottanta (Milano: Mursia, I990); Lino Pertile, 'The Italian Novel Today: Politics, Language, Literature', in The New Italian Novel, a cura di Zygmunt Barański e Lino Pertile (Edinburgh: Edinburgh University Press, I993), pp. I-I9; Andrea Cortellessa, 'Gaddismo mediato. "Funzioni Gadda" negli ultimi dieci anni di narrativa italiana', Allegoria, X.28 (I998), 4I-78.

2 Enrico Testa, Lo stile semplice. Discorso e romanzo (Torino: Einaudi, I997), p. 8.

3 Vittorio Coletti, Storia dellitaliano letterario. Dalle origini al Novecento (Torino: Einaudi, I993), p. 385 . Sulla stessa falsariga: Filippo La Porta, 'Gli ultimi vent'anni: nuovi romanzi per nuovi lettori', in Carlo Madrignani et al., 'La narrativa', in Manuale di letteratura italiana: storia per generi e problemi, a cura di Sandro Brioschi e Costanzo Di Girolamo, vol. Iv, Dall'unità d'Italia alla fine del Novecento (Torino: Bollati Boringhieri, I996), pp. 6I6-34 (p. 630); Walter Pedullà, Carlo Emilio Gadda. Lo scrittore come delinquente (Milano: Rizzoli, I997), pp. I4-I 5.

4 Il capofila è Pier Vittorio Tondelli (Correggio, I955-9I) di Altri libertini e Pao pao (Milano: Feltrinelli, I980; I982), ma si pensi anche alle prime prove della tondelliana Silvia Ballestra (Porto San Giorgio, I969), come Il compleanno dell'iguana e Il disastro degli Antò (Ancona: Transeuropa, I99I; I992). Cfr. Hermann Haller, 'Sull'uso letterario del dialetto nel romanzo recente', in Lingua e dialetto nella tradizione letteraria italiana (Roma: Salerno editrice, I996), pp. 60I-I2.

5 La citazione è tratta da Joseph Farrell, 'Vincenzo Consolo. Metaphors and 
False History', in The New Italian Novel, pp. 59-74 (pp. 59-60).

6 Alessandro Baricco (Torino, I958), Oceano mare (Milano: Rizzoli, 1993).

7 Giuseppe Ferrandino (Ischia, I958), Pericle il nero (Bologna: Granata Press, I993; poi Milano: Adelphi, I998).

8 Laura Pariani (Busto Arsizio, I95 I), D $i$ corno o d'oro (Palermo: Sellerio, I993, premi Grinzane Cavour, Piero Chiara, Città di Roma opera prima).

9 Silvana Grasso (Macchia di Giarre, I952), Nebbie di Ddraunnàra (Milano: La Tartaruga, I993, premi Grinzane Cavour e Mondello).

${ }^{\text {Io }}$ La prima recensione alla raccolta della Pariani, per esempio, è a firma di una delle più influenti talent-scout dell'epoca, Grazia Cherchi: 'Poveracci da antologia', l'Unità, 24 maggio 1993. Grasso e Pariani approdano nel tempo a case editrici maggiori quali Rizzoli, Einaudi, Marsilio, pur continuando a collaborare con editori di nicchia.

${ }^{\text {I }}$ Per un inquadramento dell'opera della Grasso si veda Sharon Wood, 'I romanzi di Silvana Grasso', in Il romanzo contemporaneo. Voci italiane, a cura di Franca Pellegrini e Elisabetta Tarantino (Leicester: Troubador, 2006), pp. 93I05, e, per un'esaustiva analisi linguistica, Marina Castiglione, L'incesto della parola. Lingua e scrittura in Silvana Grasso (Caltanissetta: Sciascia, 2009), a cui rimando anche per la bibliografia. $\mathrm{Si}$ veda inoltre l'Archivio Silvana Grasso, $<$ http://www.archiviosilvanagrasso.it/> [per questo e per gli altri siti internet, l'ultima data di consultazione è il 23 maggio 20I3]. Sull'opera della Pariani si vedano: Gigliola Sulis, 'Il racconto come militanza: sulle radici femministe dell'opera di Laura Pariani', e 'Gli anni settanta, il femminismo, l'arte. Conversazione con Laura Pariani', in 'On ne naît pas ... on le devient'. Les Gender Studies et le cas italien, des années '7o à aujourd'hui, a cura di Lisa El Ghaoui e Filippo Fonio (Grenoble: ELLUG I6, 2013), pp. 303I 5 e 316-24; Ead., 'Le migranti nell'opera di Laura Pariani, tra radicamento locale e dimensione transazionale. Una via italiana al postcoloniale?', in Narrativa, 33/34. Coloniale e postcoloniale nella letteratura italiana degli anni 2000 (20I2), a cura di Silvia Contarini et al., pp. 265-73; Claudia Nocentini, 'Laura Pariani and the Value of Experience', in The Poetics of the Margin: Mapping Europe from the Interstices, a cura di Rossella Riccobono (Oxford: Peter Lang, 20IO), pp. I33-56; Domenica Perrone, 'L'ossessione di raccontare. La narrativa di Laura Pariani', in La coscienza e il coraggio. Esperienze letterarie della modernità. Studi in onore di Sandro Maxia, a cura di Giovanna Caltagirone (Cagliari: AM\&D, 2005), pp. 809-2I; Brigitte Urbani, 'Laura Pariani: Dio non ama $i$ bambini (2007) o la storia di un giovane serial killer nell'Argentina del secolo scorso', in Il romanzo poliziesco. La storia, la memoria, a cura di Claudio Milanesi (Bologna: Astræa, 2009), pp. 49-66; Ead., 'Tra passato e presente. Scrittura femminile di Laura Pariani', in Narrativa, 30. Maschile/femminile nella letteratura italiana degli anni 2000 (2008), pp. III-23; Ead., 'Du Lago d'Orta à la Tierra del Fuego. Les personnages des récits de Laura Pariani', in Les habitants du récit. Voyage critique dans la littérature italienne des années soixantedix à nos jours, a cura di Denis Ferraris (Paris: Presses de la Sorbonne Nouvelle, 2008), pp. 77-96; Giuseppe Mazzocchi, 'La spada e la luna di Laura Pariani: una scrittrice italiana e la cultura ispanica', Italica, LXXIV (I997), 375-9I. Il sito web della scrittrice contiene molti materiali, incluse accurate sezioni bibliografiche e un archivio delle recensioni: <http:// www.omegna.net/pariani>.

I2 Di preferenza, si utilizzeranno strumenti critici in sintonia con tale clima o a esso riconducibili, in linea con il percorso intellettuale della scrittrice e nel rispetto della specificità della riflessione e delle pratiche culturali italiane di fine Novecento.

I3 Maria Corti, 'Dialetti in appello' [1963 $\left.{ }^{\mathrm{I}}\right]$, in Ead., Metodi e fantasmi (Milano: Feltrinelli, I969), pp. I09-I7 (pp. II 5-I6). Il fatto che l'invito della 
Corti si riveli ancora valido a distanza di cinquant'anni mi pare indicativo della lentezza negli studi di lingua e stile degli scrittori secondo-novecenteschi e della contemporaneità.

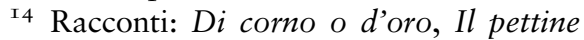
(Palermo: Sellerio, I993; I995), La perfezione degli elastici (e del cinema) (Milano: Rizzoli, I997), Il paese delle vocali (Bellinzona: Casagrande, 2000), L'uovo di Gertrudina (Milano: Rizzoli, 2003), Il paese dei sogni perduti. Anni e storie argentine e Patagonia Blues (Milano: Effigie, 2004 e 2006), I pesci nel letto (Padova: Alet, 2006), Ghiacciofuoco (con Nicola Lecca, Venezia: Marsilio, 2006). Romanzi: La spada e la luna (Palermo: Sellerio, I995), La Signora dei porci, La foto di Orta, Quando Dio ballava il tango, La straduzione (Milano: Rizzoli, rispettivamente I999; 2001; 2002; 2004), Tango per una rosa (Bellinzona: Casagrande, 2005), Dio non ama i bambini, Milano è una selva oscura, La valle delle donne lupo (Torino: Einaudi, 2007; 2010; 20II), Le montagne di Don Patagonia (Novara: Interlinea, 20I2), Il piatto dell'angelo (Milano: Giunti, 20I3). La pubblicistica è disseminata in rubriche e interventi per molte testate italiane, tra cui La Stampa, Avvenire, Il secolo XIX, Il Corriere della Sera, Il Sole 24 Ore, Diario.

I5 Il primo risale nel I966, con la madre, sulle tracce del nonno che, emigrato negli anni venti, si era creato una seconda famiglia in Sudamerica (cfr. $I l$ piatto dell'angelo).

${ }^{16}$ Parte del lavoro di ricerca compiuto sugli emigrati italiani in Argentina è ripreso in Laura Pariani, Il paese dei sogni perduti. Anni e storie argentine, e, con particolare riferimento alle questioni linguistico-letterarie, in ' $\mathrm{La}$ lingua dei Tanos e la letteratura argentina', in Into and Out of Italy. The Language and Culture of Migrants, a cura di Anna Laura Lepschy et al. (Perugia: Guerra, 20I0), pp. I49-52. La scrittrice si occupa inoltre di questioni argentine nella pubblicistica, e contribuisce a promuovere la conoscenza degli scrittori sudamericani in Italia grazie al sito <www.narratori. org $>$. Nel 2004 ha tradotto per Einaudi il romanzo $\grave{E}$ sempre difficile tornare a casa dell'italo-argentino Antonio Dal Masetto (ed. or. I985).

${ }^{17}$ Cfr. 'Di corno o d'oro', nella raccolta omonima, pp. 9-54 (p. 9). Un episodio sudamericano compare pure, incassato come ricordo rievocato dal personaggio della 'Scrittrice', nelle otto 'Notti' che costituiscono la cornice de La Signora dei porci.

${ }^{18} \mathrm{Si}$ veda la Milano 'parola magica' ed evocatrice di distanza estrema ne $I l$ paese delle vocali, p. I4 e Il piatto dell'angelo, p. ıо, o meta agognata di una liceale di provincia nel racconto 'Liberté, Egalité, Marrons Glacés', in La storia siamo noi. Quattordici scrittori raccontano l'Italia dal I 848 a oggi, a cura di Mattia Carratello (Vicenza: Neri Pozza, 2008), pp. I7 I-202.

${ }^{19}$ Cfr. Michail Bachtin, 'La parola in Dostoevskij', in Dostoevskij. Poetica e stilistica $\left[\mathrm{I} 963^{\mathrm{I}}\right]$, trad. it. Giuseppe Garritano (Torino: Einaudi, I968), pp. 235-352.

${ }^{20}$ Es., in Homi Bhabha, 'Culture's InBetween', in Multicultural States. Rethinking Difference and Identity, a cura di David Bennett (London: Routledge, I989), pp. 29-36.

2I Adriana Cavarero, A più voci. Filosofia dell'espressione vocale (Milano: Feltrinelli, 20IO), pp. 2IO-II; cfr. Hannah Arendt, Vita activa. La condizione umana [1958 $\left.{ }^{\mathrm{I}}\right]$ (Milano: Bompiani, I989), p. I90. Il riferimento a Hannah Arendt mi sembra congruo con la formazione della scrittrice e con il suo orizzonte culturale, specialmente se proposto nella lettura in chiave femminista della Cavarero, filosofa della differenza di genere, co-fondatrice della Libreria delle Donne a Milano (I975) e della comunità filosofica Diotima a Verona (I983). Sulle opportunità e i rischi connessi all'estensione al contesto italiano di categorie critiche quali quelle del postcoloniale, quando 
questa non sia sorretta da una ricognizione della riflessione critica endogena, mi sono espressa in 'Le migranti nell'opera di Laura Pariani', p. 273.

${ }^{22}$ Cfr. le dichiarazioni di poetica in ' $I l$ nostro niente che reclama amore. Intervista a Laura Pariani', a cura di Paolo Di Paolo, Milano, 5 marzo 2004, nella sezione 'Interviste' del sito Italia Libri, <http://www.italialibri.net/inter viste/040I-5.html >: 'Sono affettivamente legata al dialetto che sento come lingua materna, lingua dell'appartenenza al territorio in cui sono nata: da qui il mio tentativo di rifarmi alla sua secolare tradizione ricca di espressività carnosa, che purtroppo è stata negli ultimi anni cancellata, trasformata, travisata, svenduta come folclore $o$ banalità da barzelletta. Penso però che la letteratura del Novecento, soprattutto in poesia, abbia mostrato pienamente le potenzialità ancora racchiuse in queste lingue tradizionali che stanno scomparendo'.

${ }^{23}$ Si vedano le indicazioni fornite in Laura Pariani, 'Tre noticine', in Milano è una selva oscura, p. I79; cfr. Carlo Porta, Poesie, a cura di Dante Isella, nuova edizione rivista e accresciuta (Milano: Mondadori, 2000 [I975 $\left.\left.{ }^{\mathrm{I}}\right]\right)$, pp. 226-4I, 672-96; Carlo Righetti (alias Cletto Arrighi), Dizionario milanese-italiano - col repertorio italiano-milanese (Milano: Hoepli, I896). Per un panorama della tradizione letteraria lombarda, si veda Dante Isella, I lombardi in rivolta. Da Carlo Maria Maggi a Carlo Emilio Gadda (Torino: Einaudi, I984).

${ }^{24}$ Per un esempio della frequenza di proverbi e conte dialettali si veda, ad apertura di pagina, e per difetto, $L a$ Signora dei porci, pp. 19, 26, 28, 30, 43, 50-5I, 56, 60, 6I, 7I, 73, 78, 88, 90, 98, 99, го0, І03, I19, І24, І33, I44, I 5I, I60, I6I, 248. Cfr. anche, con plurilinguismo implicito, Quando Dio ballava il tango, cit., p. 300: 'la voce della cantante racconta il dolore di una creatura nata quando Dio voltava la testa dall'altra parte; uno dei tanti modi di dire dialettali che gli emigrati si sono portati dietro in Argentina dall'Italia'. Nel romanzo, ogni capitolo si apre con la citazione di una canzone argentina, in traduzione italiana.

25 A una commistione dialettal-argentina si deve anche il nome del protagonista di Milano è una selva oscura, Dante, detto 'lingéra' non solo per una forma di un realismo linguistico (il termine significa 'barbone' in milanese), ma, come scopre chi si addentra nel macrotesto dell'opera dalla scrittrice, anche in omaggio a Francisco Rìmoli (1903-38), poeta anarchico argentino e autore di testi per tanghi, che assunse lo pseudonimo di Dante A. Linyera in onore di Dante e dei vagabondi, in lunfardo 'linyera' (a sua volta derivato circolarmente dal lombardo). Il vocabolo è particolarmente caro alla scrittrice, che lo utilizza già nel romanzo La straduzione, ambientato a Buenos Aires e incentrato sugli anni argentini dello scrittore polacco Witold Gombrowicz: 'Lynieras, vagabondi [...] Cani sciolti' (Pariani, La straduzione, pp. 38-39).

26 Poche le eccezioni, come alcuni racconti, es. 'I nottambuli', in Laura Pariani, La perfezione degli elastici (e del cinema), pp. 47-68, e il romanzo breve Tango per una rosa, in cui compaiono pochi inserti in francese, spagnolo, inglese, legati alla vita internazionale del protagonista, l'aviatorescrittore Antoine de Saint-Exupéry.

${ }^{27}$ Il 'bagulûn dul lüstar' è la figura dell'imbonitore, che ingannava i contadini alle fiere.

${ }^{28}$ Per il lombardo, cfr. ad vocem il Vocabolario Milanese-Italiano, a cura di Francesco Cherubini (Milano: Dall'Imp. Regia stamperia, I839-56), 5 voll.

29 Cfr. Michail Bachtin, 'La parola nel romanzo' [1934-35 ${ }^{\mathrm{I}}$, in Id., Estetica e romanzo, traduzione di Clara Strada Janovič (Torino: Einaudi, I979), pp. 67-230 (pp. 79-80).

30 Bachtin, Dostoevskij, p. I3.

${ }^{31}$ Che della vita delle donne non rimanga traccia è una consapevolezza di genere 
espressa nel romanzo dal 'Primo coro delle donne': 'Na vita senza storia, compagna a quella di chi vi ha precedute, fino a quando verrà ul Miedidùr e allora ognuna di voi la andarà a durmì senza che nessuno la disturbi' (La Signora dei porci, p. 90).

${ }^{2} \mathrm{Si}$ vedano le pagine conclusive de $\mathrm{La}$ Signora dei porci, in cui la Sanguèta, sotto tortura, offre alla misteriosa divinità adorata dalle donne, la Signora della Prea-crüa, il racconto della propria vita, in cambio del suo aiuto per una buona morte: “[...] vi conterò la mé storia". La Signora sorride soddisfatta: "Brava. È la risposta giusta"; dato che a Lei non importa che la si preghi o le si diano offerte, ma solo le basta che qualcuno le parli: perché alla Signora piace sentir contare storie' (p. 252). Domenica Perrone individua nell'inesausta declinazione del dolore e dell'offesa' il centro propulsore dell'opera della Pariani ('L'ossessione di raccontare', p. 809).

33 Sia Cora e Venturina che la 'sciura milanese' e Fenísia parlano al caldo di una cucina, mentre la stanza della 'Scrittrice' ospita la conversazione con il 'Mietitore'; per tutte, le conversazioni avvengono la notte.

34 Pariani, racconto omonimo in L'uovo di Gertrudina, pp. 213-18 (p. 218).

35 Nocentini, 'Laura Pariani and the Value of Experience', p. I33, con citazioni dai saggi di Italo Calvino, 'Usi politici giusti e sbagliati della letteratura' [1976 ${ }^{\mathrm{I}}$, in Id., Saggi. I945-1985, a cura di Mario Barenghi (Milano: Mondadori, I995), pp. 350-60, e Umberto Eco, 'Il gioco dell'occupazione' [I968 ${ }^{\mathrm{I}}$ ], in Id., Il costume di casa (Milano: Bompiani, I973), pp. 299-302.

36 Prova a contrario è l'aumentato grado di difficoltà di lettura ne La foto d'Orta, di cui è protagonista Friedrich Nietzsche, che ritorna con la memoria a una gita sul lago (presso il quale risiede ora la Pariani). Nei frequenti soliloqui del personaggio l'italiano interagisce con il tedesco, che per un lettore italofono, a differenza del lombardo o dello spagnolo, non è decifrabile per vie intuitive.

37 Gayatri C. Spivak, 'Can the Subaltern Speak?', in Marxism and the Interpretation of Culture, a cura $\mathrm{di}$ Cary Nelson e Lawrence Grossberg (Basingstoke: Macmillan, I988), pp. 27I-3I3, poi ampliato in Ead., $A$ Critic of Postcolonial Reason: Toward a History of the Vanishing Present (Cambridge, MA: Harvard University Press, I999), da cui si cita; trad. it. Critica della ragione postcoloniale (Roma: Meltemi, 2004).

${ }^{8}$ Secondo la Spivak, per l'intellettuale occidentale 'the Other as Subject is unaccessible': 'the colonized subaltern subject is irretrievably heterogeneous' (A Critic of Postcolonial Reason, pp. 268 e 270). L'ansia dei subaltern studies di investigare, identificare e misurare ciò che rende specifico il soggetto colonizzato rivelerebbe un programma essenzialista che, nell'oggettificare l'Altro come gruppo monolitico, ne annulla le differenze interne (p. 270; p. 284 sulla costruzione della 'monolithic third-world woman').

39 Con la consueta consapevolezza, sul passaggio dall'oggetto del racconto al soggetto che lo racconta riflette la voce narrante nella cornice de La straduzione, costruita con evidenti connotati autobiografici, che, attraverso lo schermo della storia di Gombrowicz, descrive al figlio Luca i luoghi della sua Buenos Aires: 'Mi faccio domande sul senso di tutto questo, di ogni vita, forse. [...] E quale senso ho io, seduta davanti a una finestra; quale importanza hanno gli scontri con mia madre, ora che lei non c'è più, se io adesso non li rievoco con qualcuno? Con te. L'unica cosa che ha senso è il raccontare' (La straduzione, p. 46). Cfr. anche, ne 'L'uovo di Gertrudina', p. 218: 'mi pare a volte che tutti i miei personaggi siano racchiusi nella stessa storia, la mia'.

$4 \circ$ Sulla rilevanza della Milano degli anni Settanta per la scrittrice, cfr. Sulis, 'Il racconto come militanza: sulle radici femministe dell'opera di Laura Pariani' 
e 'Gli anni settanta, il femminismo, l'arte'. Sul vivace fronte del movimento delle donne e del femminismo nella Milano dell'epoca, cfr. Judith Adler Hellman, Journeys among Women. Feminism in Five Italian Cities (Cambridge: Polity Press, I987), pp. 8I-IIO.

${ }^{4}$ I Un dialogo a distanza (per es. la lettera al figlio ne La straduzione o l'allocuzione alla madre scomparsa ne Il piatto dell'angelo) o in presentia (per es. l'intervista, registrata e trascritta, in Quando Dio ballava il tango e La valle delle donne lupo, il colloquio con l'ultimo interlocutore di ogni vita, il 'Mietitore', ne La Signora dei porci).

${ }^{42} \mathrm{La}$ ricerca della parola poetica come azione collettiva diviene momento narrativo ne $L a$ straduzione, in cui gli amici del bar uniscono le forze per tradurre il romanzo Ferdydurke di Gombrowicz in castigliano dal polacco, lingua che per lo più non conoscono (cfr. La straduzione, pp. I66-69, I80$85)$.

43 Cavarero, A più voci, p. 2 Iо.

${ }^{44} \mathrm{Si}$ veda il volume collettivo del 'Gruppo per l'espressione della donna', Perché non $i$ fiori (Milano: La Salamandra/ CELUC [Cooperativa Editrice Libraria Università Cattolica], I975), e La fata rovesciata (Milano: Ottaviano, I976), firmato con lo pseudonimo Laura Picco. Alcune tavole sono riproposte nella sezione 'Fumetti' del sito della scrittrice.

45 La protagonista de La fata rovesciata, di cui seguiamo la crescita dall'infanzia alla maturità, ricorda: 'ero muta, non avevo lingua né parole [...] mi propinarono la cultura del più forte, come ai poveri a cui si insegna la lingua che li emarginerà' (Laura Picco, La fata rovesciata, p. 88).

${ }^{46} \mathrm{E}$ ancora, nei pensieri della figlia Ipólita: 'A volte mi vien da dire, riflette l'Ipólita guardando il frate e il notario, che chi non ha mai vissuto in brughiera al pödi cumpréndi nagòtta di noióltar paisàn' (La Signora dei porci, p. I03). Si veda, a titolo di confronto, un testo realmente prodotto da una donna processata per stregoneria nel primo Cinquecento a Collevecchio, nella Sabina, di cui rimane sia una confessione autografa sia la trascrizione rimaneggiata dal notaio (cfr. Pietro Trifone, 'La confessione di Bellezze Ursini "strega" nella campagna romana del Cinquecento', CoFIM. Contributi di filologia dell'Italia Mediana, II (I988), 79-I 82). Sull'opposizione linguistica tra donna inquisita e inquisitori, cfr. Giuseppe Antonelli, 'Italiano degli umili, italiano dei potenti', in La lingua nella storia d'Italia, a cura di Luca Serianni (Roma: Società Dante Alighieri, 200I), pp. 547-63 (pp. 54748).

47 Sull'imposizione dell'italiano ai bambini dialettofoni nella scuola fascista, cfr. da ultimo Maria Catricalà, 'Questione della lingua e scuola nell'Italia unita', in La lingua nella storia d'Italia, a cura di Serianni, pp. 53 I-46 (pp. 537-38).

${ }^{8}$ Nei racconti di area lombarda, per esempio, si assiste anche al recupero di forme di lavoro femminile tradizionale, come l'allevamento dei bachi da seta (cfr. 'Fine del gran bigatto', in $\mathrm{D} i$ corno o d'oro, pp. 55-62).

49 Cit. in Francesco Mannoni, 'Carlo Ginzburg e i modi di scandagliare il passato', Giornale di Brescia, I 8 maggio 2007, p. 34 (corsivo mio).

50 Cfr. Luisa Passerini, Autoritratto di gruppo (Milano: Giunti, 1988).

${ }^{5}$ Cfr. Carlo Ginzburg, Il formaggio e $i$ vermi. Il cosmo secondo un mugnaio del '500 (Torino: Einaudi, I976), come anche il precedente $I$ benandanti. Ricerche sulla stregoneria e sui culti agrari tra Cinquecento e Seicento (Torino: Einaudi, I966), sulla società contadina friulana e sul suo sincretismo religioso, o al successivo volume dedicato alla stregoneria, Storia notturna. Una decifrazione del Sabba (Torino: Einaudi, I989). Si noti come attorno ad un processo per stregoneria contro una giovane donna, Antonia, a fine Cinquecento, si sviluppi anche il 
romanzo La chimera di Sebastiano Vassalli (Torino: Einaudi, I990, Premio Strega). Come ne La Signora dei porci, fortissima è la componente territoriale — la storia è ambientata a Zardino, paese ormai inesistente, in provincia di Novara (non lontano, dunque, dai luoghi della Pariani) mentre divergono il trattamento linguistico e il punto di vista adottato.

52 Intervista a Laura Pariani, a cura di Domenica Perrone. Sul ruolo ispiratore di Cesare Bermani e del suo lavoro di raccolta di testimonianze orali, oltre che sulle interviste condotte in prima persona, si veda la 'Nota dell'autrice' ne La valle delle donne lupo, p. 24I.

53 Sulis, 'Gli anni settanta, il femminismo, l'arte'.

54 Cfr. Maria Bellonci, Lucrezia Borgia e Rinascimento privato (Milano: Mondadori, I939; I985), citata dalla Pariani nel dibattito di "On ne naît pas... on le devient". I gender studies e il caso italiano, dagli anni Settanta a oggi, tra liberazione sessuale e nuovi tabì, ottavo convegno internazionale organizzato dal GERCI (Groupe d'études et de recherches sur la culture italienne), Université Stendhal-Grenoble 3 (Grenoble, 25-26 novembre 2010).

55 Cfr. l'intervista rilasciata in occasione della presentazione de La valle delle donne lupo al programma radiofonico 'Farheneit', RadioTre Rai, 23 febbraio 2OI2, ascoltabile in podcast nel sito $<$ www.radio3.rai.it>. Cfr. Armanda Guiducci, La donna non è gente (Milano: Rizzoli, I977). Sulla Guiducci, cfr. Sharon Wood, Italian Women's Writing. I860-I994 (London: The Athlone Press, 1995), p. 194, e da ultimo Frances J. Parmeggiani, 'Armanda Guiducci e le sfide dell'identità', in 'On ne naît pas ... on le devient', pp. 27 I-80.

${ }^{56}$ Cfr. Wood, Italian Women's Writing, pp. I85-274 (p. 2I4), e Carole LazzaroWeiss, From Margins to Mainstream. Feminism and Fictional Modes in Italian Women's Writing, I968-1990 (Philadelphia: University of Pennsylvania Press, I993). Nella letteratura di consumo, si pensi al successo di Susanna Tamaro con Va' dove ti porta il cuore (Milano: Rizzoli, I994).

57 Cfr. Sulis, 'Gli anni settanta, il femminismo, l'arte'. Sulle articolazioni del femminismo italiano, cfr. Hellman, Journeys among Women; Italian Feminist Theory and Practice. Equality and Sexual Difference, a cura di Graziella Parati e Rebecca West (Madison: Fairleigh Dickinson University Press, 2002); e Le filosofie femministe. Due secoli di battaglie teoriche e pratiche, a cura di Adriana Cavarero e Franco Restaino (Milano: Bruno Mondadori, 2002).

${ }^{8}$ Anna Maria Ortese, L'Iguana (Firenze: Vallecchi, I965). Riprendo la lettura del romanzo in chiave di allegoria coloniale dal saggio di Daniela La Penna, 'An Inquiry into Modality and Genre: ReConsidering L'Iguana by Anna Maria Ortese' in The Italian Gothic and Fantastic: Encounters and Rewritings of Narrative Traditions, a cura di Francesca Billiani e Gigliola Sulis (Madison: Fairleigh Dickinson University Press, 2007), pp. I60-87, da cui traggo anche la seguente dichiarazione della Ortese (p. I77): 'Posso dire d'essere mossa a lavorare dall'indignazione. Anche la matrice del racconto lungo L'Iguana [...], sta tutta lì. Io sento molta indignazione e molto dolore per la condizione del povero. A me sembra che chi non ha denaro, cioè potere, oggi non esiste. Questa condizione di non esistenza riporta l'uomo in grembo alla natura. Ecco: a Milano c'è tanta bontà, ma c'è anche tanta chiusura mentale. Per questo ho immaginato un giovane editore milanese alle prese con L'Iguana che rappresenta gli esseri umani dei Paesi non industrializzati. Non hanno denaro, e così sono anche grotteschi, ridicoli'.

59 Le storie lombarde sono quasi del tutto assenti dalle traduzioni. Le prime trasposizioni sono in tedesco, con i racconti de Il pettine e i romanzi La spada e la luna, ambientato tra il Perù e la Spagna, nel secondo Cinquecento, e La foto di Orta. Oggi, la scrittrice è tradotta soprattutto in francese (Il 
paese delle vocali, Quando Dio ballava il tango, Tango per una rosa, Dio non ama $i$ bambini, oltre ad alcuni racconti) e in spagnolo (Quando Dio ballava il tango, Dio non ama $i$ bambini). La straduzione, omaggio a Gombrowicz, è stato riproposto in polacco, e pochi racconti sono disponbili in olandese e arabo. In inglese, cfr. 'Night Owls' ('I nottambuli'), trad. di Martha King, in After the War (New York: Italica Press, 2004), pp. I39-62 e 'The Color Black Has a Huge Mouth' ('Il colore nero ha una bocca immensa'), trad. di Jamie Richards, in Word Without Borders. The Online Magazine for International Literature, $<\mathrm{http} / /$ wordswithoutborders.org/article/thecolor-black-has-a-huge-mouth $>$. Per i dati bibliografici si veda la sezione 'Traduzioni' del sito della scrittrice.

${ }^{60} \mathrm{La}$ Pariani si divide da sempre tra pittura, fumetti e racconto, e non di rado gli stimoli della pittrice divengono pietra focaia per la narrazione, i cui personaggi e le cui atmosfere traggono spunto da un dettaglio visivo (un'immagine, una foto, un dipinto). Tale processo creativo è spesso tematizzato, alla maniera di Tabucchi, a volte sin dal titolo, come nel romanzo La foto d'Orta. Alcuni esempi: la foto del nonno emigrato ne Il piatto dell'angelo; per Milano è una selva oscura, le 'fotografie di Marco Mandibola che ritraggono un barbone milanese della fine degli anni Sessanta' ('Tre noticine'); una sezione di fotografie d'epoca, 'La memoria illustrata', è inserita in quanto pregresso visivo delle testimonianze raccolte in Il paese dei sogni perduti, pp. 65-93, come pure ne Le montagne di Don Patagonia; per La Signora dei porci, la scrittrice cita la suggestione emanante, sin dall'infanzia, da un affresco di una chiesa del paese natale: 'una torre, sulla cima della quale danza una donna circondata da porci' (Intervista a Laura Pariani, a cura di Domenica Perrone, I6 aprile 2003, disponibile nel sito Lo specchio di carta. Osservatorio sul romanzo italiano contemporaneo, nella sezione 'Autori' $>$ 'Laura Pariani' > 'Intervista', <http:// lospecchiodicarta.unipa.it $>$ ).

${ }^{6 r}$ Cfr. Enzo Siciliano, Racconti italiani del Novecento, 3 voll. (Milano: Mondadori, 200I), vol. III, pp. II952 I7 (p. II95).

${ }^{62}$ Gianfranco Contini, 'Introduzione alla Cognizione del dolore' [1963 ${ }^{\mathrm{I}}$ ], in Id., Varianti e altra linguistica. Una raccolta di saggi (I938-I968) (Torino: Einaudi, I970), pp. 60I-20. Si noti come già Contini specifichi, in chiusura, che Gadda è un unicum che non fa scuola.

${ }^{63}$ Cfr. Alfredo Stussi, Lingua, dialetto $e$ letteratura (Torino: Einaudi, I993), p. 25: 'Contini, [...] insisteva soprattutto sulle modalità soggettive nell'impiego del dialetto e, prospettando un'ossimorica "tradizione della soggettività" (altro non è la cosiddetta "linea Gadda"), restringeva l'area del vero o presunto realismo dialettale. [...] Come spesso capita, una giusta esigenza ha portato a qualche esagerazione, per esempio nell'uso indiscriminato di "espressionismo" fino ad inglobarvi talvolta tutt'intero il plurilinguismo'. Si veda anche Pier Vincenzo Mengaldo, 'Preliminari al dopo Contini' (I990), in Id., La tradizione del Novecento. Terza serie (Torino: Einaudi, I99I), pp. I6073 (p. I7I).

64 Alcuni nomi, oltre i già citati Grasso e Ferrandino: i sardi Salvatore Niffoi (Orani, I950-), Sergio Atzeni (Capoterra, 1952-Carloforte, I995) e Marcello Fois (Nuoro, I960-); Giancarlo De Cataldo (Taranto, I956), pugliese trapiantato a Roma, città di cui subisce il fascino linguistico, come avvenne già a Gadda e Pasolini; il napoletano Giuseppe Montesano (Napoli, I959-); l'emiliano Carlo Lucarelli (Parma, I960-).

${ }^{65}$ Cfr. Mario Chiesa e Giovanni Tesio, Il dialetto da lingua della realtà a lingua della poesia (Torino: Paravia, I978).

${ }^{66}$ In questa direzione si muove, per esempio, la raccolta di autobiografie 
linguistiche di autori siciliani proposta da Marina Castiglione in

'Dal plurilinguismo domestico al plurilinguismo letterario. Casi di studio in Sicilia', The Italianist, $32 \cdot 3$ (20I 2), 32I-44.

\section{Notes ON CONTRIBUTOR}

Gigliola Sulis is Lecturer and Director of Italian at the University of Leeds. Her research interests include multilingualism in Italian literature, the language and style of contemporary Italian writers, the literary canon, modern dialect poetry, Sardinian literature, and twentieth-century women's writing. Her research approach integrates stylistics, narratology, and issues of identity raised by multicultural diversity.

Correspondence to: Gigliola Sulis. Email: g.sulis@leeds.ac.uk 